\title{
Artérite à virus du cheval : enquête séro-épidémiologique dans deux régions de la Tunisie
}

\author{
A. Chabchoub ${ }^{1 *}$ F. Landolsi ${ }^{1}$ L. Mkaouer ${ }^{1}$ \\ F. Lasfar ${ }^{2}$ A. Ghorbel ${ }^{1}$ A. Ghram ${ }^{3}$
}

Mots-clés

Equidae - Cheval Pur-sang Arabe Cheval Barbe - Artérite - Virose Epidémiologie - Tunisie.

\begin{abstract}
Résumé
Une enquête séro-épidémiologique sur l'artérite à virus des équidés a été réalisée dans deux régions de la Tunisie : I'Ariana et Kasserine. Les auteurs ont recherché par la technique de séroneutralisation les anticorps spécifiques dirigés contre le virus de l'artérite dans un échantillon représentatif de l'effectif équin dans les deux régions. Cet échantillon a concerné 199 chevaux dont 114 ont été des Pur-sang Arabes provenant de la région de l'Ariana et 85 des Barbes de la région de Kasserine ; 72 ont été des mâles et 127 des femelles. Les résultats ont montré que 51 chevaux (25,6 p. 100) ont été positifs, parmi lesquels 30 (26,3 p. 100) ont été des Pur-sang Arabes de la région de l'Ariana et 21 (24,7 p. 100) des chevaux Barbes de la région de Kasserine ; la différence $\mathrm{n}^{\prime}$ a pas été statistiquement significative. Douze mâles (16,66 p. 100) et 39 femelles (30,7 p. 100) ont été positifs ; la différence a été statistiquement significative. Ces résultats sont discutés en relation avec ceux obtenus par $d^{\prime}$ autres auteurs en Tunisie et dans les pays voisins.
\end{abstract}

\section{INTRODUCTION}

L'artérite à virus est une maladie contagieuse spécifique des équidés. Elle induit souvent des avortements en série dans un élevage et entraîne des pertes économiques considérables (3, 23, 24, 26, 27). Le virus de l'artérite équine a été isolé pour la première fois en 1953, suite à une épizootie d'avortements et de troubles respiratoires chez des chevaux à Bucyrus (Ohio, Etats-Unis) (26). En 1984 une épizootie d'artérite est survenue dans l'Etat du Ken-

\footnotetext{
1. Service de pathologie médicale des équidés et carnivores, Ecole nationale de médecine vétérinaire, Sidi-Thabet 2020, Tunisie

2. Fondation nationale d'amélioration de la race chevaline, Sidi-Thabet 2020 , Tunisie

3. Laboratoire de microbiologie vétérinaire, Institut Pasteur de Tunis, BP 74, 13 Place Pasteur, Belvedère, Tunisie

* Auteur pour la correspondance

Tél. : +21671552200; fax : +21671552441

E-mail : chabchoubahmed2001@yahoo.fr ou chabchoub.ahmed@iresa.agrinet.tn
}

tucky, aux Etats-Unis et, depuis, le nombre de foyers déclarés a considérablement augmenté dans le monde (12, 13, 16, 20, 21, 25).

En Tunisie, des enquêtes séro-épidémiologiques ont montré la circulation à bas bruit de l'artérite à virus dans l'effectif équin $(5,8$, 14). De plus, des cas d'avortements dont l'origine n'a pas été identifiée avec certitude ont été signalés chez la jument. Ainsi, compte tenu de la gravité de l'infection et du manque d'informations actualisées sur la situation épidémiologique de cette maladie, les auteurs ont réalisé, au cours de l'année 2000, une enquête séro-épidémiologique dont l'objectif a été d'évaluer le taux d'infection de l'artérite virale chez les chevaux Pur-sang Arabes et Barbes dans deux régions de la Tunisie : l'Ariana et Kasserine.

MATERIEL ET METHODES

\section{Matériel \\ Population enquêtée}

La population équine en Tunisie est relativement importante. D'après les statistiques, elle compte 255270 équidés dont 37000 
chevaux. Ces derniers sont composés de 2000 chevaux Pur-sang Arabes, 1500 chevaux Pur-sang Anglais, 900 chevaux Barbes, le reste de la population étant des dérivés du Barbe. Dans cet effectif, on distingue des chevaux de course, de selle et des chevaux destinés à la reproduction (7). Ce sont généralement les chevaux Pursang Arabes et Pur-sang Anglais. Ils sont bien entretenus, vivent dans de bonnes conditions d'hygiène et font l'objet d'un suivi médical permanent. On trouve également les chevaux de travail utilisés pour effectuer la traction et les travaux agricoles en milieu rural. Ce sont généralement des chevaux Barbes, Arabes-Barbes ou d'origine indéterminée. Ils appartiennent à des élevages traditionnels où les conditions d'hygiène et sanitaires sont souvent passables. Cette population équine est inégalement répartie sur le territoire tunisien.

La race Barbe se rencontre dans l'ouest de la Tunisie, en zone frontalière avec l'Algérie, au pied de l'Atlas, et dans les plaines limitrophes de Kasserine et du Kef, chez les tribus de Fréchiche et d'Ouerdène. Ces régions représentent la première zone où l'on trouve le plus grand nombre de chevaux Barbes. La deuxième zone est représentée par le centre de la Tunisie, surtout la région de Kairouan où il $\mathrm{y}$ a la tribu de Zlass.

Le cheval Pur-sang Arabe est concentré surtout dans certaines régions du nord du pays. Ce sont les régions où existent des infrastructures nationales, comme les haras, les clubs hippiques ou les champs de courses. Le Grand Tunis, particulièrement la région de l'Ariana, du fait de la proximité du haras de Sidi-Thabet et des champs de course de Ksar Said, représente la zone de plus forte concentration du cheval Pur-sang Arabe.

Ces caractéristiques géographiques de la population équine en Tunisie ont amené les auteurs à choisir la région de l'Ariana, située au nord-est, et la région de Kasserine, située au centre-ouest, pour réaliser l'enquête épidémiologique. Ces deux régions sont respectivement représentatives de la population des chevaux Pursang Arabes et des chevaux Barbes.

\section{Echantillon}

L'échantillon a été obtenu par tirage au sort stratifié sur la région. La répartition géographique des chevaux testés a été similaire à la répartition des populations selon la race et le mode d'élevage. En effet, dans la région de l'Ariana on trouve les chevaux de race Pursang Arabe vivant dans des élevages bien entretenus et faisant l'objet d'un suivi médical et sanitaire régulier. En revanche, dans la région de Kasserine, les chevaux sont en majorité de race Barbe ou dérivée, ils sont élevés dans des conditions d'entretien et d'hygiène passables et ils ne font pas systématiquement l'objet d'un suivi médical et sanitaire. La population cible a été divisée en deux strates homogènes. A l'intérieur de chaque strate, un tirage au sort des chevaux à tester a été effectué à partir d'une liste établie par la Fondation nationale d'amélioration de la race chevaline (Fnarc).

\section{Taille de l'échantillon}

La taille de l'échantillon a été déterminée en fonction de l'effectif des chevaux dans chaque strate, de la prévalence pré-estimée de l'infection et du degré de précision choisi (22). L'effectif des chevaux étudiés a été de 860 animaux dont 620 chevaux de race Pursang Arabe dans la région de l'Ariana et 240 chevaux Barbes dans la région de Kasserine.

La prévalence pré-estimée a été définie à partir d'enquêtes séroépidémiologiques réalisées antérieurement qui ont rapporté des taux d'infection ayant varié entre 8 p. 100 (14) et 30 p. 100 (8) et qui n'ont pas révélé de différences statistiquement significatives entre les races et le mode d'élevage. Ceci a permis de retenir une prévalence pré-estimée de 20 p. 100 pour les deux strates étudiées. Les auteurs ont aussi fixé une précision de 33 p. 100.

\section{Nombre d'animaux tirés au sort}

Parmi les 199 chevaux tirés au sort, 85 étaient dans la région de Kasserine et 114 dans la région de l'Ariana. Leur âge a varié entre 2 et 23 ans avec une moyenne de 9,97 \pm 4,61 ans. Il y avait 72 mâles et 127 femelles.

\section{Méthodes}

Le dépistage de l'infection a été réalisé par la technique de séroneutralisation en microplaque sur cellules Vero en monocouche au laboratoire de virologie de l'Institut Pasteur de Tunis. C'est une méthode de référence largement utilisée pour le diagnostic de l'artérite virale équine $(1,17,26,27)$. Elle détecte les immunoglobulines $\mathrm{G}(\mathrm{IgG})$ associées à la neutralisation du virus de l'artérite équine, qui représentent les témoins d'une immunité acquise due à une infection ancienne (26). Les cellules Vero constituent une lignée cellulaire continue douée d'un potentiel illimité de croissance. Ce sont des cellules épithéliales de reins de singe utilisées en couche unique pour l'isolement et l'identification du virus. Elles ont été fournies par l'American Type Cell Collection (Atcc) et conservées dans de l'azote liquide. La souche Bucyrus du virus de l'artérite équine utilisée a été gracieusement fournie par le Docteur Zientara de l'Agence française de sécurité sanitaire des aliments (Afssa). La séroneutralisation permet la détection des anticorps spécifiques dirigés contre le virus de l'artérite équine dans les sérums d'équidés prélevés. Un sérum est positif quand il inhibe l'effet cytopathique du virus à une dilution supérieure au 1/10 (10, 11, 18).

Pour l'étude statistique, ont été calculés la moyenne (p), l'écarttype (ET) et l'intervalle de confiance (IC) pour chaque paramètre. Les résultats ont été présentés en retenant : IC $=p \pm 2 \mathrm{ET}$. La comparaison entre les groupes a été réalisée par le test du $\mathrm{Chi}^{2}$ et par le calcul de l'écart réduit (e). La différence a été considérée comme significative au risque d'erreur de 5 p. 100 (22).

\section{RESULTATS}

Sur les 199 chevaux testés, 51 se sont révélés positifs (25,62 p. 100). L'intervalle de confiance a été compris entre 19,44 et 31,8 p. 100. Dans la région de l'Ariana, 30 chevaux Pur-sang Arabes sur 114 se sont révélés positifs (26,31 p. 100). Dans la région de Kasserine 21 chevaux Barbes sur 85 se sont révélés positifs ( 24,7 p. 100). Il n'y a pas eu de différence statistiquement significative entre les deux régions pour le taux de positivité.

Sur les 51 sérums positifs, 25 ont eu des titres sériques en anticorps neutralisant positifs au 1/20, 15 des titres positifs au 1/40, 4 des titres positifs au $1 / 80$ et 7 des titres positifs au 1/160. Parmi les 72 mâles, 12 se sont révélés positifs, soit un taux de positivité de 16,66 p. 100. Chez les 127 femelles, 39 ont été positives, soit un taux de positivité de 30,70 p. 100. La différence entre le taux de positivité des mâles et des femelles a été statistiquement significative $\left(\mathrm{Chi}^{2}=4,73\right)$.

En considérant l'âge, les auteurs ont noté que les animaux de moins de quatre ans ont été négatifs (tableau I). La comparaison deux à deux des taux de positivité entre les différents intervalles a révélé des différences significatives (e > 1,96) entre les différentes tranches d'âges (tableau II). Les animaux âgés de 8-12 ans ont eu un taux de positivité statistiquement supérieur à celui des animaux âgés de 12-16 ans. 
Tableau I

Nombre de sérums testés et de sérums positifs envers l'artérite à virus en fonction de l'âge des chevaux étudiés

\begin{tabular}{lccc} 
Age & Nb. de sérums testés & Nb. de sérums positifs & Taux de positivité $(\%)$ \\
\hline$<4$ ans & 12 & 0 & - \\
$4-8$ ans & 49 & 10 & 20,4 \\
$8-12$ ans & 71 & 25 & 35,21 \\
$12-16$ ans & 36 & 6 & 16,66 \\
$>16$ ans & 31 & 10 & 32,25 \\
Total & 199 & 51 & 25,62
\end{tabular}

\section{Tableau II}

Comparaison deux à deux des pourcentages de séropositivité envers l'artérite à virus par le calcul de l'écart réduit en fonction de l'âge des chevaux étudiés

\begin{tabular}{|c|c|c|c|c|c|}
\hline & $<4$ ans & 4-8 ans & 8-12 ans & $12-16$ ans & $>16$ ans \\
\hline$<4$ ans & & 3,54 & 6,21 & 2,68 & 3,84 \\
\hline $4-8$ ans & & & 1,83 & 0,436 & 1,16 \\
\hline $8-12$ ans & & & & 2,2 & 0,29 \\
\hline $12-16$ ans & & & & & 1,84 \\
\hline$>16$ ans & & & & & \\
\hline
\end{tabular}

La différence est significative si e > 1,96

\section{DISCUSSION}

Ce travail a révélé un taux global de séropositivité de 25,62 p. 100. Ces résultats sont comparables à ceux déjà obtenus en Tunisie en 1980 par Ellouze (8) qui a trouvé un taux de positivité de 30 p. 100 sur 137 équidés testés. Ces résultats demeurent cependant supérieurs à ceux obtenus par Choura en 1986 (5) et Ibn Amor en 1993 (14) qui ont révélé des séroprévalences respectives de 9,95 p. 100, avec un effectif de 201 chevaux, et de 8,75 p. 100, avec un effectif de 400 chevaux .

En ce qui concerne les titres sériques d'anticorps neutralisant, on constate que 78 p. 100 des chevaux testés ont eu des titres sériques faibles (inférieurs ou égaux au 40e). Ces taux de positivité à des titres assez faibles plaideraient en faveur d'une circulation ancienne de l'artérite à virus en Tunisie. En effet, certains auteurs rapportent que la réponse immunitaire varie en fonction de la souche infectante et que les titres sériques augmentent à des seuils très élevés après des infections récentes, puis diminuent rapidement après, mais persistent longtemps (9). D'ailleurs, lors de la présente enquête, les auteurs n'ont pas noté de chevaux présentant une symptomatologie caractéristique et tous les chevaux de moins de quatre ans ont été trouvés négatifs.

Les prélèvements ont concerné deux types d'élevages, les animaux issus d'élevages privés individuels ruraux (Kasserine) qui connaissent des conditions sanitaires différentes des animaux vivant dans les haras (région de l'Ariana) qui eux bénéficient de meilleures conditions avec des contrôles sanitaires et médicaux réguliers et permanents. Malgré cette différence dans les conditions d'élevage et de la race, il n'y a pas eu de différence statistiquement significative entre les deux régions d'élevage.
El Harrak et coll. en 1996 (7) et Ellouze en 1980 (8) ont noté des taux de positivité supérieurs dans les haras par rapport à ceux obtenus chez des animaux vivant individuellement. Ils ont expliqué ce fait par la grande contagiosité de l'artérite à virus qui, comme le notent Brion et coll., est une «maladie de rassemblement» (2). D'ailleurs, d'après El Harrak et coll., le pourcentage élevé de séropositivité dans un haras peut être la cause, comme il peut être le résultat, d'une extension de la maladie dans la région où il est situé (7). Il n'y a pas eu de différence statistiquement significative entre les deux races, ce qui permet d'avancer qu'il n'y a pas de prédisposition de races à l'artérite équine.

Les présents résultats ne sont pas en accord avec ceux trouvés par Ghram et coll. en 1994 (11) en Tunisie ainsi que par Moraillon et coll. en 1978 au Maroc (19). Ces derniers remarquent, en effet, l'existence d'une corrélation positive entre la race et l'atteinte par le virus de l'artérite équine avec un taux d'infection chez les Barbes et les Arabes Barbes supérieur à celui observé chez les chevaux Pursang.

Dans cette étude, une différence statistiquement significative a été notée entre les résultats obtenus chez les chevaux en fonction du sexe. Ces résultats concordent avec ceux d'El Harrak et coll. (7) qui ont noté, dans leur enquête au Maroc en 1996, que les femelles sont plus infectées que les mâles, montrant ainsi l'importance de la transmission vénérienne qui est exclusivement du mâle vers la femelle (6, 12). En revanche, les présents résultats ne sont pas en accord avec ceux de Moraillon et Moraillon en 1978 (18), et de Zientara et coll. en 1998 (26) qui n'ont pas trouvé de différences statistiquement significatives entre l'atteinte par le virus des mâles et des femelles, et avec ceux de Ghram et coll. en 1994 (11) qui ont trouvé que les mâles étaient plus touchés que les femelles. Ces derniers ont proposé 
comme explication le fait que les mâles peuvent héberger le virus de l'artérite équine dans la sphère génitale pendant plusieurs années induisant ainsi une réponse sérologique positive, sans pour autant présenter des signes cliniques, contrairement aux femelles qui éliminent rapidement le virus après l'infection.

En considérant l'âge, les chevaux âgés de moins de quatre ans se sont révélés négatifs pour l'artérite à virus. Ceci pourrait être rattaché au fait que ces jeunes chevaux n'étant pas utilisés pour la reproduction sont préservés du risque d'infection par la voie vénérienne $(7,19)$. Cette séropositivité pourrait également résulter de l'arrêt de la circulation virale en Tunisie. Néanmoins, le faible nombre de ces jeunes (12 chevaux) ne permet pas de tirer des conclusions définitives.

Pour les chevaux âgés de quatre ans et plus, des taux d'infection variables ont été constatés selon les classes d'âge, avec peu de différence significative entre ces classes. Les travaux de plusieurs auteurs $(7,15,19,21)$ rapportent une augmentation d'animaux positifs avec l'âge, ce qui ne peut être précisé dans la présente étude.

\section{- CONCLUSION}

L'enquête a montré l'existence d'un taux élevé de chevaux positifs vis-à-vis de l'artérite virale équine dans les deux régions étudiées, relativement distantes, avec un taux global de 25,62 p. 100. Ce taux a été significativement supérieur à ceux trouvés par Choura en 1986 et Ibn Amor en 1993. En revanche, il a été similaire à celui obtenu par Ellouze en 1980 laissant craindre une recrudescence de cette infection. Par ailleurs, le pourcentage d'animaux séropositifs n'a pas différé significativement en fonction de la race, du type d'élevage et de la région. Des taux de positivité élevés ont été cependant trouvés chez les femelles par rapport aux mâles et chez les animaux les plus âgés.

Néanmoins, dans les régions étudiées, les animaux n'ont pas montré de manifestations cliniques rappelant l'artérite virale équine, à l'exception de quelques avortements sporadiques. Une vigilance accrue de la part des éleveurs, des vétérinaires et des responsables des haras est donc nécessaire. Une recherche sérologique systématique devrait être entreprise, notamment lors d'avortements. De même, les prélèvements d'organes et d'avortons seraient indispensables pour la mise en évidence de l'agent causal.

La vaccination utilisant des vaccins à virus vivant ou inactivé est possible, mais elle n'est pas effectuée en Tunisie. La prophylaxie sanitaire reste le seul moyen efficace pour lutter contre cette maladie du cheval. Toutes ces dispositions conjuguées avec un renforcement des contrôles aux frontières pourraient contribuer à mieux contrôler cette virose en Tunisie.

\section{BIBLIOGRAPHIE}

1. BALASURIYA U.B.R., PATTON J.F., ROSSITO P.V., TIMONEY P.J. MCCOLLUM W.H., MCLACHLAN J., 1997. Neutralization determinant of laboratory strains and field isolates of equine arteritis virus: identification of four neutralization sites in the amino-terminal ectodon of GL enveloppe glyco-protein. Virology, 232: 114-128

2. BRION A., FONTAINE M., MORAILLON R., 1967. Fièvre typhoïde du cheval. Artérite à virus. Rec. Méd. vét., 143 : 17-27.

3. CHABCHOUB A., 1988. Contribution à l'étude de la législation en matière de vente du cheval. Maghreb Vét., $3:$ 46-49.

4. CHABCHOUB A., BOUSRIH A., 1998. Situation actuelle du cheval Barbe en Tunisie. El Baytari, 19 : 9-11.

5. CHOURA I., 1986. Contribution à l'étude de I'artérite à virus équine dans les haras de Tunisie. Thèse Doct. vét., Ecole nationale de médecine vétérinaire, Sidi-Thabet, Tunisie.

6. DE VRIES A.F., ROTTIER P.J.M., GLASER A.L., HORZINER M.C., 1996. Equine arteritis virus, virus infection of equines, In: Studdert M.J. Ed., Virus infections of vertebrates. Amsterdam, The Netherlands, Elsevier, p. 171-200.
7. EL HARRAK M., HMIDOUCHE A., CHAKRI A., OURAGH L., BAKKALIKASSIMI L., 1996. Epidémiolologie de l'artérite à virus chez les équidés au Maroc. Prat. vét. Equine, 28 : 285-292.

8. ELLOUZE M.R., 1980. Contribution à l'étude épidémiologique de l'anémie infectieuse, de l'artérite à virus, de la rhinopneumonie et de la grippe équine en Tunisie. Thèse Doct. vét., Ecole nationale de médecine vétérinaire, Sidi-Thabet. Tunisie.

9. FUKUNAGA Y., MATSUMURA T., SUGIURA T., WADA R., IMAGAWA H., KANEMARU T., KAMADA M., 1994. Use of the serum neutralisation test for equine viral arteritis with different virus strains. Vet. Rec., 134: 574-576.

10. FUKUNAGA Y., WADA R., HIRASAWA K., TABUCHI E., AKIAMA Y., 1981. Clinical and virological findings on experimental equine viral arteritis. Bull. Equine Health Res. Inst., 18: 110-118.

11. GHRAM A., CHABCHOUB A., TURKI I., BOUSSETTA M., IBN AMOR H., GHORBEL A., 1994. La rhinopneumonie et l'artérite à virus du cheval : étude séroépidémiologique dans le nord-est de la Tunisie. Arch. Inst. Pasteur Tunisie, $71:$ : 12 .

12. HOLYOAK G.R., GILE R.C., MCCOLLUM W.H., LITTLE T.V., TIMONEY P.J., 1993. Pathological changes associated with equine arteritis virus infection of reproductive tract in prepubertal and peripubertal colts. J. comp. Pathol., 109: 281-293.

13. HULLINGER P.J., GARDNER I.A., HIETALA S.K., FERRERO G.L., MCLACHLAN N.J., 2001. Seroprevalence of antibodies against equine arteritis virus in horses residing in the United States and imported horses. J. Am. vet. Med. Assoc., 219: 946-949.

14. IBN AMOR H., 1993. Enquête séro-épidémiologique sur la rhinopneumonie et l'artérite à virus du cheval en Tunisie. Thèse Doct. vét., Ecole nationale de médecine vétérinaire, Sidi-Thabet, Tunisie.

15. LAHLOU K., 1977. Enquête épidémiologique sur l'anémie infectieuse, la rhinopneumonie et l'artérite à virus des équidés au Maroc. Thése Doct. vét., IAV Hassan II, Rabat, Maroc.

16. MONREAL L., VILATORO A.J., HOOGHUIS H., ROS L., TIMONEY P., 1992. Clinical features of the 1992 outbreak of equine viral arteritis in Spain. Equine vet. J., 4: 171-178.

17. MORAILLON R., MORAILLON A., 1974. Acquisitions récentes dans l'épidémiologie de l'artérite à virus du cheval en France. Rec. Méd. vét., 150 : 1015-1021.

18. MORAILLON R., MORAILLON A., 1978. Results of an epidemiological investigation on equine viral arteritis in France and some other European and African countries. Ann. Rech. vét., 9: 43-54.

19. MORAILLON A., MORAILLON R., TOMA B., SEDRATI A., LAHLOU K., 1978. Enquête épidémiologique de l'anémie infectieuse, de l'artérite à virus, de la rhinopneumonie et de la grippe équine au Maroc. Rec. Méd. vét., 11 : 921-928.

20. NEWTON J.R., WOOD J.L.N., CASTILLO-OLIVARES F.J., MUMFORD J.A., 1999. Serological surveillance of equine viral arteritis in the United Kingdom since the outbreak in 1993. Vet. Rec., 145: 511-516.

21. OUAHMA E., 1987. Enquête épidémiologique sur l'anémie infectieuse, la rhinopneumonie et l'artérite à virus. Rec. Méd. vét., 143 : 17-27.

22. SCHWARTZ D.T., 1983. Méthodes statistiques à l'usage des médecins et des biologistes. Paris, France, Flammarion Médecine Sciences, 318 p.

23. TIMONEY P.J., MCCOLLUM W.H., 1993. Equine viral arteritis. Vet. Clin. North Am. Equine Pract., 9: 295-309.

24. TIMONEY P.J., MCCOLLUM W.H., MURPHY T.W., 1991. A longitudinal study of equine arteritis virus infection in standard-bred stallion with special reference to occurrence of the carrier state. In: Plowright W., Rossdale P.D., Wade J.F. Eds, Proc. 6th International Conference on Equine Infectious Diseases, Cambridge, UK, 1991. Newmarket, UK, R\&W Publications, p. 231.

25. TIMONEY P.J., MCCOLLUM W.H., ROBERT A.W., MCDONALD M.J., 1987. Status of equine viral arteritis in Kentucky. J. Am. vet. Med. Assoc., 191: 36-39.

26. ZIENTARA S., LABIE J., GICQUEL B., RIMLINGER F., BERNADAC M., 1998. L'artérite virale des équidés / revue et bilan d'une enquête sérologique en France de 1996 à 1997. Point vét., 29 : 247-253.

27. ZIENTARA S., VILLARINO G., SCHLOTTERER C., LABIE J., GICQUEL B., 1995. A propos d'un foyer d'artérite virale équine en France. Prat. vét. Equine, $27: 23-30$.

Reçu le 25.11.2002, accepté le 04.07.2003 


\section{Summary}

Chabchoub A., Landolsi F., Mkaouer L., Lasfar F., Ghorbel A., Ghram A. Seroepidemiological Survey on Equine Viral Arteritis in Two Regions in Tunisia

A seroepidemiological survey was carried out on equine viral arteritis in two regions of Tunisia: Ariana and Kasserine. The authors researched specific antibodies against the arteritis virus by the serum neutralization technique in a sample representative of the horse populations in both areas. The sample concerned 199 horses of which 114 were Thoroughbred Arabian from Ariana area and 85 were Barb from Kasserine area; 72 of them were males and 127 females. Results showed that 51 horses $(25.6 \%)$ were seropositive. Among them 30 (26.3\%) were Arabian horses from Ariana and $21(24.7 \%)$ were Barb horses from Kasserine; the difference was not statistically significant. Twelve males $(16.66 \%)$ and 39 females $(30.7 \%)$ were positive; the difference was statistically significant. The results are discussed in relation with those obtained by other authors in Tunisia and neighboring countries.

Key words: Equidae - Thoroughbred Arabian horse - Barb horse - Arteritis - Virosis - Epidemiology - Tunisia.

\section{Resumen}

Chabchoub A., Landolsi F., Mkaouer L., Lasfar F., Ghorbel A., Ghram A. Arteritis viral equina: encuesta seroepidemiológica en dos regiones de Túnez

Se llevó a cabo una encuesta seroepidemiológica sobre arteritis viral equina en dos regiones de Túnez: Ariana y Kasserine. Los autores buscaron, mediante la técnica de seroneutralización, los anticuerpos específicos dirigidos contra el virus de la arteritis en una muestra representativa de la cabaña equina en ambas regiones. La muestra estaba compuesta por 199 caballos: 114 purasangres árabes procedentes de la región de Ariana y 85 berberiscos de la región de Kasserine; 72 eran machos y 127 hembras. Los resultados pusieron de manifiesto que 51 caballos $(25,6 \%)$ fueron positivos, 30 de los cuales $(26,3 \%)$ eran purasangres árabes de la región de Ariana y 21 $(24,7 \%)$ caballos berberiscos de la región de Kasserine. La diferencia no fue estadísticamente significativa. Doce machos $(16,66 \%)$ y 39 hembras $(30,7 \%)$ fueron positivos; la diferencia fue estadísticamente significativa. Se examinan dichos resultados comparándolos con los que otros autores obtuvieron en Túnez y en países vecinos.

Palabras clave: Equidae - Caballo purasangre árab - Caballo berberisco - Arteritis - Virosis - Epidemiología - Túnez. 